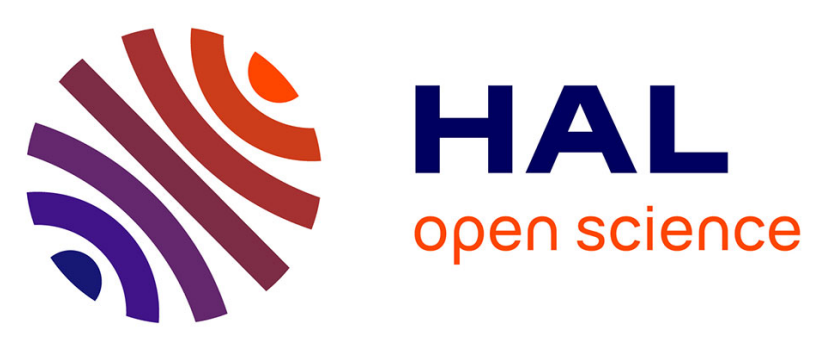

\title{
Étude en réflexion de la diffraction d'une onde lumineuse par une onde de rayleigh en tenant compte de l'influence des couches voisines de la surface
}

\author{
C. Bruneel, P. Lenglart, J. Pouliquen
}

\section{- To cite this version:}

C. Bruneel, P. Lenglart, J. Pouliquen. Étude en réflexion de la diffraction d'une onde lumineuse par une onde de rayleigh en tenant compte de l'influence des couches voisines de la surface. Revue de Physique Appliquée, 1973, 8 (4), pp.311-314. 10.1051/rphysap:0197300804031100 . jpa-00243688

\section{HAL Id: jpa-00243688 \\ https://hal.science/jpa-00243688}

Submitted on 1 Jan 1973

HAL is a multi-disciplinary open access archive for the deposit and dissemination of scientific research documents, whether they are published or not. The documents may come from teaching and research institutions in France or abroad, or from public or private research centers.
L'archive ouverte pluridisciplinaire HAL, est destinée au dépôt et à la diffusion de documents scientifiques de niveau recherche, publiés ou non, émanant des établissements d'enseignement et de recherche français ou étrangers, des laboratoires publics ou privés. 


\title{
ÉTUDE EN RÉFLEXION DE LA DIFFRACTION D'UNE ONDE LUMINEUSE PAR UNE ONDE DE RAYLEIGH EN TENANT COMPTE DE L'INFLUENCE DES COUCHES VOISINES DE LA SURFACE
}

\author{
C. BRUNEEL, P. LENGLART $(*)$ et J. POULIQUEN $(* *)$ \\ Laboratoire d'Ultrasons, I. S. E. N., 3, rue F. Baës, 59046 Lille Cedex, France
}

(Reçu le 23 février 1973, révisé le 14 mai 1973)

\begin{abstract}
Résumé. - Nous considérons l'influence conjuguée de l'ondulation de la surface, de la variation de l'indice de réfraction en surface liée à l'onde de pression et de la réflexion optique sur les couches internes provoquée par l'atténuation en profondeur de l'onde de Rayleigh.

Une application numérique permet d'évaluer l'ordre de grandeur de la contribution relative de chacun de ces phénomènes.

Abstract. - In the study of the reflective diffraction of an optic wave by a Rayleigh wave, we take into account not only the undulation of the surface but also the variation of the refractive index of the surface and the optical reflection from the interior of the medium owing to the Rayleigh wave attenuation in depth.

A numerical calculation gives us the order of magnitude of the relative contribution of each effect.
\end{abstract}

Divers auteurs [1] à [5] ont traité la diffraction en réflexion d'un faisceau lumineux par une onde de Rayleigh en tenant compte uniquement de l'ondulation de la surface créée par l'onde acoustique. Toutefois d'autres effets moins importants liés à la variation de l'indice de réfraction interviennent et sont en général négligés sans aucune évaluation. Nous analysons ici ces phénomènes et évaluons leur influence respective dans le cas d'un verre d'indice $n=1,55$ et en lui assignant comme coefficients photoélastiques la moyenne de ceux du quartz [7] $\left(P_{11}=0,12, P_{12}=0,25\right)$. Ceci semble suffisant pour obtenir l'ordre de grandeur des diverses contributions. Le choix d'un faisceau lumineux uniforme est justifié par le fait qu'il donne pour les faisceaux diffractés les mêmes amplitudes qu'un faisceau gaussien [3]. Dans un article précédent [6] résumé ici nous avions déjà étudié la modulation d'amplitude de l'onde réfléchie due à la variation $\mathrm{du}$ coefficient de réflexion de la surface ( $1^{\text {re }}$ correction). Il reste à préciser l'importance des réflexions à l'intérieur du matériau provoquées par le gradient de l'indice de réfraction lié à l'atténuation de l'onde acoustique avec la profondeur ( $2^{\mathrm{e}}$ correction).

Les composantes d'une onde de Rayleigh se propa-

(*) Laboratoire de Physique des Solides, ISEN, 3, rue François-Baës, 59046 Lille Cedex. Equipe de recherche du CNRS.

$(* *)$ Laboratoire d'Ultra-Sons de la Faculté Libre des Sciences de Lille et du GEFIRN, 3, rue François-Baës, 59046 Lille Cedex. Equipe de recherche associée au CNRS. geant dans la direction $x$ pour le choix d'axes de la figure 1, ont pour expression [8] :

$$
\begin{aligned}
& U_{x}=\left[\exp \left(k \alpha_{l} z\right)-\beta \alpha_{t} \exp \left(k \alpha_{t} z\right)\right] a \cos (\Omega t-k x) \\
& U_{z}=\left[\exp \left(k \alpha_{t} z\right)-\alpha_{l} \exp \left(k \alpha_{l} z\right)\right] a \sin (\Omega t-k x)
\end{aligned}
$$

où les coefficients $\alpha_{l}, \alpha_{t}, \beta$ caractéristiques de l'onde de Rayleigh sont liés au coefficient de Poisson $\sigma$ [9] $\mathrm{du}$ milieu. Dans le cas du verre on a : $\sigma=0,24$, $\alpha_{l}=0,84, \alpha_{t}=0,40, \beta=1,46$. « $a$ » est une constante proportionnelle à l'amplitude de l'onde de Rayleigh, $k$ et $\Omega$ sont le vecteur d'onde et la pulsation de cette onde.

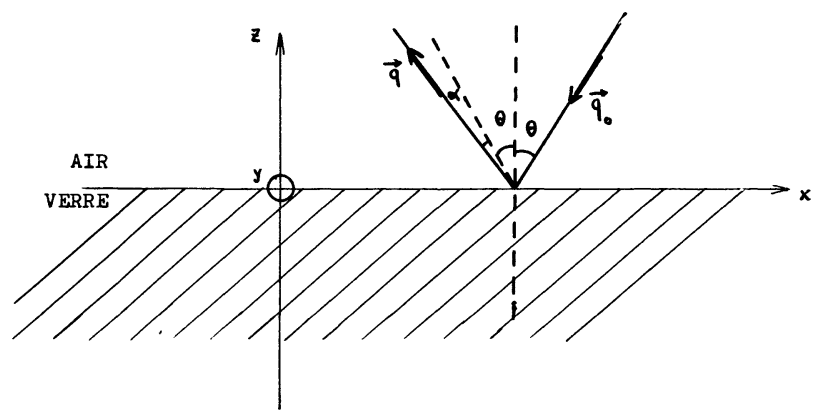

Fig. 1. - Réflexion en surface.

L'équation de l'ellipsoïde des indices s'écrit :

$$
\begin{aligned}
a_{11} X^{2} & +a_{22} Y^{2}+a_{33} Z^{2} \\
& +2 a_{12} X Y+2 a_{13} X Z+2 a_{23} Y Z=1
\end{aligned}
$$

où $X, Y$ et $Z$ sont les composantes de l'indice selon les axes du trièdre de référence. 
Des éq. (1) on peut obtenir le tenseur des déformations $\left(S_{i j}\right)$ qui donne les variations des coefficients $a_{i j}$ de l'ellipsoïde des indices [10] :

$$
\begin{aligned}
\delta a_{11}=a_{11}-a_{11}^{0}= & P_{11} S_{11}+P_{12} S_{33}= \\
= & 0,022 a k \sin (\Omega t-k x) \\
\delta a_{22}=a_{22}-a_{22}^{0}= & P_{12} S_{11}+P_{12} S_{33}= \\
= & 0,078 a k \sin (\Omega t-k x) \\
\delta a_{33}=a_{33}-a_{33}^{0}= & P_{12} S_{11}+P_{11} S_{33}= \\
= & 0,089 a k \sin (\Omega t-k x)
\end{aligned}
$$

avec

et

$$
a_{11}^{0}=a_{22}^{0}=a_{33}^{0}=\frac{1}{n_{0}^{2}}
$$

$$
\begin{aligned}
& a_{13}=\left(\frac{P_{11}-P_{12}}{2}\right) S_{13}=0,0011 a k \cos (\Omega t-k x) \\
& a_{12}=a_{13}=0 .
\end{aligned}
$$

Dans la suite $a_{13}$ faible devant les autres termes sera négligé. Il entraînerait une légère rotation de l'ellipsoïde des indices perpendiculairement à la direction $O y$.

$\delta a_{i i}$ est faible car « $a$ » mesuré optiquement est de l'ordre de quelques dizaines d'angströms [11] et $\Lambda=2 \pi / k$ peut varier du micron au millimètre. En se limitant au premier ordre, on obtient :

$$
\delta a_{i i}=-\frac{2 \delta n_{i i}}{n_{i i}^{3}}
$$

et

$$
\delta n_{i i}=C_{i i} \sin (\Omega t-k x)
$$

avec

$$
\begin{gathered}
C_{11}=-0,041 a k, \quad C_{22}=-0,144 a k, \\
C_{33}=-0,167 a k
\end{gathered}
$$

et pour une direction quelconque on pore :

$$
\delta n=C \sin (\Omega t-k x) \text { avec } C=F\left(C_{i i}\right) .
$$

1. Contribution à la raie diffractée de la réflexion sur la surface. - (Nous reprenons succinctement un article antérieurement publié) [6].

Considérons figure 1 une onde lumineuse polarisée de vecteur d'onde $\mathbf{q}_{0}$ (perpendiculaire à $O y$, ce qui conserve la symétrie de translation suivant cet axe), d'amplitude $A_{0}$ et de pulsation $\omega$, elle donne après réflexion sur la surface, dans la direction $\mathbf{q}$ une onde lumineuse déterminée par :

$$
A(\mathbf{q})=\frac{A_{0}}{2 b} \int R(\mathbf{r}) \exp i\left[\omega t+\mathbf{r}\left(\mathbf{q}_{0}-\mathbf{q}\right)\right] \mathrm{d} x
$$

où le coefficient de réflexion en amplitude $R(\mathbf{r})$ a la forme :

$$
R(\mathbf{r})=R_{0}+\frac{\mathrm{d} R}{\mathrm{~d} n} \delta n(\mathbf{r})
$$

et $\mathbf{r}\left(x, y, U_{z}\right)$ : vecteur position du point réfléchissant.
Après intégration sur une largeur $2 b$ du faisceau et sachant que :

$$
\exp (i u \sin \varphi)=\sum_{-\infty}^{+\infty} \exp (i m \varphi) J_{m}(u)
$$

avec $J_{m}(u)$ fonction de Bessel d'ordre $m$; on obtient :

$$
\begin{aligned}
A(\alpha) & =A_{0} \exp (i \omega t)\left[R_{0} \sum_{m} \exp (i m \Omega t) J_{m}(d) f(m) \pm\right. \\
& \left. \pm \frac{\mathrm{d} R}{\mathrm{~d} n} \frac{C}{2 i} \sum_{m} \exp [i(m \pm 1) \Omega t] J_{m}(d) f(m \pm 1)\right]
\end{aligned}
$$

où

$$
d=4 \pi\left(\beta-\alpha_{l}\right) \frac{a}{\lambda} \cos \left(\theta+\frac{\alpha}{2}\right) \cos \frac{\alpha}{2},
$$

$\theta$ angle d'incidence, $(\theta+\alpha)$ angle de réflexion,

$$
\begin{gathered}
f(m)=\frac{\sin [X(m)]}{X(m)}, \\
X(m)=\frac{2 \pi b}{\lambda}\left[2 \sin \frac{\alpha}{2} \cos \left(\theta+\frac{\alpha}{2}\right)-m \frac{\lambda}{\Lambda}\right]
\end{gathered}
$$

$\lambda$ et $\Lambda$ représentent les longueurs d'onde optique et acoustique.

Ceci correspond à un spectre de raies et avecl'approximation $a / \lambda$ faible, un développement au premier ordre en $a / \lambda$ des fonctions de Bessel donne :

$$
\begin{gathered}
A(\alpha=0)=A_{0} R_{0} \\
A_{ \pm 1}=A_{0}\left[R_{0} \frac{d}{2} \pm \frac{\mathrm{d} R}{\mathrm{~d} n} \frac{C}{2 i}\right] .
\end{gathered}
$$

Les raies latérales font avec la raie centrale un angle $\alpha$ tel que $2 \sin \alpha / 2 \cos \theta= \pm \lambda / \Lambda$. Le premier terme de ces raies $(A M P)$ (terme principal) est dû à la modulation de phase et le second $(A M A)$ à la modulation d'amplitude (1 ${ }^{\text {re }}$ correction).

Si $\theta$ et $\alpha$ sont petits

$$
\frac{1}{R_{0}} \frac{\mathrm{d} R}{\mathrm{~d} n} \simeq 1,43
$$

on obtient pour une onde polarisée parallèlement au plan d'incidence :

$$
\left(A M P_{\|} \pm i A M A_{\|}\right)=A_{0} R_{0} a\left[0,64 \frac{2 \pi}{\lambda} \pm i 0,030 \frac{2 \pi}{\Lambda}\right]
$$

et une onde polarisée perpendiculairement au plan d'incidence :

$$
\left(A M P_{\perp} \pm i A M A_{\perp}\right)=A_{0} R_{0} a\left[0,64 \frac{2 \pi}{\lambda} \pm i 0,103 \frac{2 \pi}{\Lambda}\right] \text {. }
$$

2. Contribution de la réflexion sur les couches internes. - On admet que les variations d'indice ne modifient que le pouvoir réflecteur. Pour le calcul des chemins optiques, au sein du matériau soumis aux ondes de Rayleigh, l'hypothèse de rayons lumineux se propageant en ligne droite et de $n(x, z) \simeq n_{0}$ sera conservée. 
Le retard de phase du rayon réfléchi au point $(x, z)$ par rapport au rayon réfléchi à l'origine est donné (Fig. 2) par :

$$
\varphi(x, z)=-\frac{2 \pi}{\lambda}\left[2 x \sin \frac{\alpha}{2} \cos \left(\theta+\frac{\alpha}{2}\right)+\gamma_{z}\right]
$$

où

$$
\begin{aligned}
\gamma=-n_{0}\left[\frac{1}{\cos \theta^{\prime}}+\frac{1}{\cos \left(\theta^{\prime}+\alpha^{\prime}\right)}\right]+ \\
\quad+\operatorname{tg} \theta^{\prime} \sin \theta+\operatorname{tg}\left(\theta^{\prime}+\alpha^{\prime}\right) \sin (\theta+\alpha) .
\end{aligned}
$$

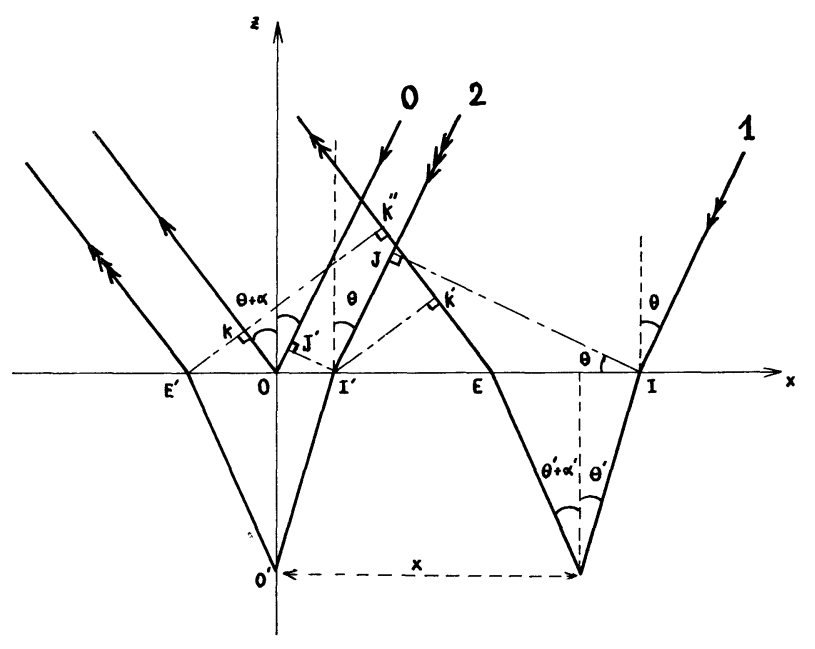

FIG. 2. - Phase des rayons réfléchis sur les couches internes

$$
\begin{aligned}
& \delta[(1)-(0)]=\delta[(1)-(2)]+\delta[(2)-(0)] \\
& \delta[(1)-(2)]=\left(E K^{\prime \prime}\right)-\left(I^{\prime} J\right) \\
& \delta[(2)-(0)]=\left(I^{\prime} O^{\prime}\right)+\left(O^{\prime} E^{\prime}\right)-\left(O J^{\prime}\right)-(O K) .
\end{aligned}
$$

Les déformations étant faibles, on limitera les calculs aux développements au premier ordre. L'indice relatif $n^{\prime}(x, z)$ d'une couche par rapport à la voisine pour un rayon se propageant dans le milieu sous un angle d'incidence $\theta^{\prime}$ (Fig. 3) est égal à :

$$
n^{\prime}(x, z)=\frac{n(x+\delta x, z+\delta z)}{n(x, z)}
$$

soit au premier ordre et compte tenu que $\delta x=\delta z \operatorname{tg} \theta^{\prime}$ :

$$
n^{\prime}(x, z)=1+\frac{1}{n_{0}}\left[\frac{\partial n}{\partial x} \operatorname{tg} \theta^{\prime}+\frac{\partial n}{\partial z}\right] \delta z
$$

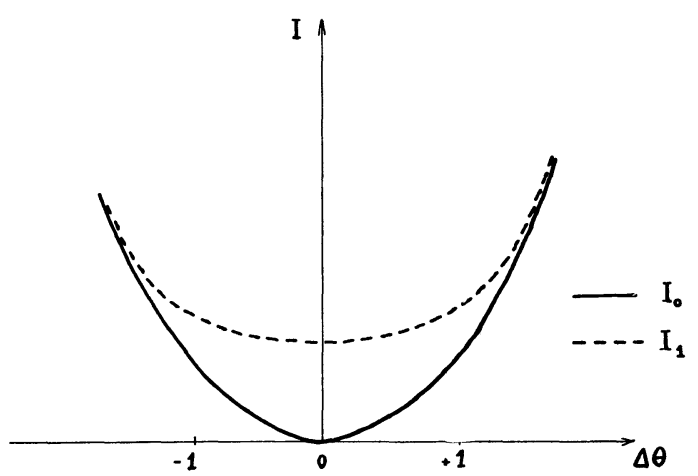

Fig. 3. - Allure de l'évolution relative des intensités de la raie diffractée d'ordre $1: I_{1}$ et de la raie de réflexion spéculaire $I_{0}$ au voisinage de l'angle de Brewster.
Le coefficient de réflexion en amplitude au point de coordonnées $(x, z)$ produit par une couche d'épaisseur $\delta z$ vaut :

$$
R(x, z)=\left.\frac{\mathrm{d} R\left(n^{\prime}\right)}{\mathrm{d} n^{\prime}}\right|_{n^{\prime}=1} \cdot \frac{1}{n_{0}}\left[\frac{\partial n}{\partial x} \operatorname{tg} \theta^{\prime}+\frac{\partial n}{\partial z}\right] \delta z
$$

puisque $R\left(n^{\prime}=1\right) \equiv 0, n(x, z)$ est de la forme :

$n(x) z=,n_{0}+\left[f_{t} \exp \left(k \alpha_{t} z\right)+f_{l} \exp \left(k \alpha_{l} z\right)\right] \times$

$$
\times a k \sin (\Omega t-k x)
$$

où $f_{t}$ et $f_{l}$ sont déterminés par les relations donnant $S_{i j}$ et les relations (3) et (4).

Pour une onde polarisée perpendiculairement au plan d'incidence $\delta n=\delta n_{22}$ quel que soit $\theta$.

Pour une onde polarisée parallèlement au plan d'incidence et tombant sous l'incidence $\theta$, l'éq. (2) donne :

$$
\delta n=\cos ^{2} \theta^{\prime} \delta n_{11}+\sin ^{2} \theta^{\prime} \delta n_{33} .
$$

Dans l'approximation des petites variations, nous pouvons calculer la contribution de chaque terme que nous sommerons ensuite. Après intégration sur toute la largeur du faisceau (en tenant compte du décalage de celui-ci) et sur une épaisseur infinie, nous obtenons deux raies latérales dans les directions des raies précédentes du $1^{\text {er }}$ ordre :

$$
A^{\prime}=\left.\frac{A_{0}}{2} \frac{a k^{2} t_{1} t_{2}}{n_{0}} \frac{\mathrm{d} R\left(n^{\prime}\right)}{\mathrm{d} n^{\prime}}\right|_{n^{\prime}=1} \times
$$

$$
\times \sum_{j=l . t} \frac{\mp i f_{j} \alpha_{i}+f_{j} \operatorname{tg} \theta^{\prime}}{k \alpha_{j}-i\left(\frac{2 \pi}{\lambda} \gamma-Y_{ \pm 1} \operatorname{tg} \theta^{\prime}\right)} \frac{\sin \left(b Y_{ \pm 1}\right)}{b Y_{ \pm 1}}
$$

où

$$
Y_{ \pm 1}=\frac{4 \pi}{\lambda} \sin \frac{\alpha}{2} \cos \left(\theta+\frac{\alpha}{2}\right) \mp k
$$

$t_{1}$ et $t_{2}$ sont les coefficients de transmission air-verre et verre-air. L'équation (12) donne pour $Y_{ \pm 1}=0$

$$
\begin{aligned}
A_{ \pm 1}^{\prime}=\frac{A_{0}}{2} \frac{a k^{2} t_{1} t_{2}}{n_{0}} & \left.\frac{\mathrm{d} R\left(n^{\prime}\right)}{\mathrm{d} n^{\prime}}\right|_{n^{\prime}=1} \times \\
& \times \sum_{j=l, t} \mp \frac{i f_{j} \alpha_{j}+f_{j} \operatorname{tg} \theta^{\prime}}{k \alpha_{j}-\frac{2 \pi}{\lambda} \gamma} .
\end{aligned}
$$

En général $\Lambda=2 \pi / k \gg \lambda$ donc

$$
k \alpha_{j}-\frac{2 \pi i \gamma}{\lambda} \simeq-\frac{2 \pi i \gamma}{\lambda}
$$

et la relation (13) devient :

où

$$
A_{ \pm 1}^{\prime}=(A R I)+\left(A^{\prime} R I\right)
$$

$$
\begin{aligned}
& A R I=\left.\frac{A_{0}}{2} \frac{a k^{2} t_{1} t_{2}}{n_{0}} \frac{\mathrm{d} R\left(n^{\prime}\right)}{\mathrm{d} n^{\prime}}\right|_{n^{\prime}=1} \sum_{j=l, t} \frac{ \pm f_{j} \alpha_{j}}{\frac{2 \pi}{\lambda} \gamma} \\
& A^{\prime} R I=\left.\frac{A_{0}}{2} \frac{a k^{2} t_{1} t_{2}}{n_{0}} \frac{\mathrm{d} R\left(n^{\prime}\right)}{\mathrm{d} n^{\prime}}\right|_{n^{\prime}=1} \sum_{j=l, t} \frac{\mp i f_{j} \operatorname{tg} \theta^{\prime}}{\frac{2 \pi}{\lambda} \gamma} .
\end{aligned}
$$


Les termes $(A R I)$ et $(A M P)$ sont en quadrature de phase avec les termes $\left(A^{\prime} R I\right)$ et $(A M A)$.

Pour des angles $\theta$ et $\alpha$ faibles ce qui n'est plus tout à fait le cas pour $\alpha$ lorsque la fréquence est de l'ordre du gigahertz, le tableau indique les rapports des amplitudes diffractées dues aux corrections à l'amplitude de diffraction principale : $A M P$ (amplitude diffractée due à la modulation de phase), $A M A$ (1 $1^{\text {re }}$ correction, amplitude diffractée due à la modulation d'amplitude de l'onde réfléchie provoquée par la modulation du pouvoir réflecteur en surface), $A R I$ ( $2^{\mathrm{e}}$ correction, amplitude diffractée due au pouvoir réflecteur des couches internes voisines de la surface perturbée par l'onde acoustique).

Les lumières polarisées perpendiculairement et parallèlement au plan d'incidence sont caractérisées respectivement par les symboles $\perp, / /$.

A $17 \mathrm{MHz}$ la longueur d'onde acoustique $\Lambda$ vaut $0,185 \mathrm{~mm}$ et la lumière utilisée est celle d'un laser He-Ne $(\lambda=0,64 \mu)$.

\section{TABLEAU}

Rapport des diverses contributions à la raie diffractée

$\begin{array}{cccc}\frac{F}{A} & 17 \mathrm{MHz} & 340 \mathrm{MHz} & 1,7 \mathrm{GHz} \\ \frac{A M A_{\perp}}{A M P_{\perp}} & 0,6 \times 10^{-3} & 1,2 \times 10^{-2} & 6 \times 10^{-2} \\ \frac{A M A_{\|}}{A M P_{\|}} & 0,2 \times 10^{-3} & 0,4 \times 10^{-2} & 2 \times 10^{-2} \\ \frac{A R I_{\|}}{A M P_{\|}} & 0,85 \times 10^{-7} & 3,4 \times 10^{-5} & 0,85 \times 10^{-3} \\ \frac{A R I_{\perp}}{A M P_{\perp}} & 0,31 \times 10^{-6} & 1,24 \times 10^{-4} & 0,31 \times 10^{-2} \\ \frac{A^{\prime} R I_{\|}}{A M P_{\|} \operatorname{tg} \theta^{\prime}} & 0,98 \times 10^{-7} & 3,92 \times 10^{-5} & 0,98 \times 10^{-3} \\ \frac{A^{\prime} R I_{\perp}}{A M P_{\perp} \operatorname{tg} \theta^{\prime}} & 0,36 \times 10^{-6} & 1,4 \times 10^{-4} & 0,36 \times 10^{-2}\end{array}$

Ce tableau montre qu'aux basses fréquences il est parfaitement justifié de négliger les effets de variation d'indice. La correction la plus importante $(A M A)$ reste inférieure au millième du terme principal $(A M P)$. Les corrections augmentent avec la fréquence mais restent faibles. A $1,7 \mathrm{GHz}$ le terme $A M A_{\perp}$ atteint $6 \%$ du terme principal, ce qui n'est pas négligeable mais il ne faut pas oublier qu'il est en quadrature avec lui ce qui minimise la modification de l'amplitude résultante. Quant au terme $A R I_{\|}$en phase avec le terme principal il reste de l'ordre du centième de ce dernier.

On remarque, éq. (6), que $A M P$ dépend de $R_{0}$ qui s'annule pour une lumière polarisée parallèlement sous incidence de Brewster. Ceci pourrait permettre la mise en évidence des autres contributions. Expérimentalement on devrait observer aux environs de $300 \mathrm{MHz}$, pour une incidence voisine de l'angle de Brewster, les variations d'intensité lumineuse de la raie diffractée $I_{1}$ et de la raie de réflexion spéculaire $I_{0}$, proportionnelle à $R_{0}$ (Fig. 3). On a porté les intensités $I_{1}$ et $I_{0}$ en fonction de $\Delta \theta$, écart par rapport à l'angle de Brewster. $I_{1}$ et $I_{0}$ sont représentées à des échelles différentes telles que les courbes coïncident pour un angle très différent de l'angle de Brewster.

Cette expérience permettrait donc de déterminer $A M A$ et $A M P$. Puisque $A M P$ n'est fonction que de la composante transversale de l'onde de Rayleigh et que $A M A$ dépend à la fois des composantes transversales et longitudinales, ceci donnerait une vérification de la relation entre ces deux composantes.

3. Conclusion. - Cette étude justifie l'approximation courante qui ne tient compte que de l'ondulation de la surface dans la description de l'interaction d'une onde lumineuse et d'une onde de Rayleigh pour des matériaux présentant des propriétés optiques voisines de celles du verre et des fréquences basses. En se plaçant sous incidence de Brewster et à des fréquences élevées (au-dessus de $300 \mathrm{MHz}$ ), on devrait pouvoir mettre en évidence les contributions de ces effets et obtenir ainsi de nouveaux renseignements sur l'onde de Rayleigh et sur les constantes élastiques et photoélastiques en surface.

Comme les corrections sont proportionnelles aux coefficients photoélastiques, elles deviendraient non négligeables aux hautes fréquences pour des matériaux dont ces coefficients seraient plusieurs fois supérieurs à ceux du verre. Dans le cas d'une onde acoustique de surface dont le vecteur déplacement est dans le plan de la surface et perpendiculaire au vecteur de propagation (onde de Love), cette étude fait pressentir la possibilité de détection optique d'une telle onde mais avec des amplitudes diffractées comparables aux valeurs des corrections obtenues ici.

\section{Bibliographie}

[1] Mayer, W. G., Lamers, G. B. and Auth, D. C., J. Acoust. Soc. Am. 42 (1967) 1255.

[2] Hallermeier, R. J. et Mayer, W. G., J. Appl. Phys. 41 (1970) 3664.

[3] Hallermeier, R. J. et Mayer, W. G., J. Acoust. Soc. Am. 47 (1970) 1236.

[4] Lean, E. G. H. et Powell, C. G., Proc. of IEEE 58 n$^{\circ} 12$ (1970) 1939.

[5] Neighbors, T. H. et Mayer, W. G., J. Appl. Phys. 42 (1971) 3670.
[6] Bruneel, C., Lenglart, P. et Pouliouen, J. F., $C$. $R$ Hebd. Séan. Acad. Sci. Paris 273B (1971) 706-709.

[7] American Institute of Physics Handbook, 6-194.

[8] Landau, L. D. et LifSChifT, E. M., Theory of Elasticity, 105.

[9] Viktorov, I. A., Rayleigh and Lamb Waves.

[10] CADY, Piezoelectricity (1946) 713.

[11] BRUNEel, C., Rapport DEA, Orsay, septembre 1970 (non publié). 